\title{
PERIPHERAL AND CENTRAL FATIGUE AFTER HIGH INTENSITY RESISTANCE CIRCUIT TRAINING
}

\author{
GONZALO MÁRQUEZ, PhD, ${ }^{1}$ SALVADOR ROMERO-ARENAS, PhD, ${ }^{1}$ CRISTIAN MARÍN-PAGÁN, MSc, ${ }^{2}$ \\ ANTONIO VERA-IBAÑEZ, MSc, ${ }^{1}$ MIGUEL FERNÁNDEZ DEL OLMO, PhD, ${ }^{3}$ and WOLFGANG TAUBE, $\mathrm{PhD}^{4}$ \\ ${ }^{1}$ Department of Physical Education and Sport, Faculty of Sport Sciences, Catholic University of Murcia (UCAM), Murcia, Spain \\ ${ }^{2}$ Research Center for High Performance Sport. Catholic University of Murcia (UCAM), Murcia, Spain \\ ${ }^{3}$ Learning and Human Movement Control Group, Department of Physical Education and Sport, Faculty Sport Sciences and Physical \\ Activity, University of A Coruña, A Coruña, Spain \\ ${ }^{4}$ Department of Medicine, Movement and Sports Science, University of Fribourg, Fribourg, Switzerland \\ Accepted 4 November 2016
}

\begin{abstract}
Introduction: The aim of this study was to investigate the effects of high intensity resistance circuit (HIRC) and traditional strength training (TST) on neuromuscular fatigue and metabolic responses. Methods: Twelve trained young subjects performed HIRC and TST in a counterbalanced order with 1 week rest in-between. The amount of workload and the interset time for each local muscle group were matched $(180 \mathrm{~s})$, however, the time between successive exercises differed. The twitch interpolation technique was used to test neuromuscular function of the knee extensor muscles. Blood lactate concentration was used to evaluate metabolic responses. Results: Maximum voluntary contraction and resting potentiated twitch amplitude $\left(Q_{\mathrm{tw}}\right)$ were significantly reduced after HIRC, but there were not changes after TST, while reductions in voluntary activation were similar. Lactate concentration increased significantly more after HIRC. Conclusions: The higher lactate concentration after HIRC probably impaired excitation-contraction coupling, indicating larger peripheral fatigue than after TST.
\end{abstract}

Muscle Nerve 000:000-000, 2016

Resistance training is an excellent method to enhance muscular hypertrophy, strength, power, and local muscular endurance. Although the physiological mechanisms underlying these changes remain unclear, disturbances in homeostasis associated with acute muscular fatigue could be the foundation of the strength adaptations. ${ }^{1}$ Therefore, muscular fatigue induced by a bout of resistance exercise could account for long-term muscular adaptations. ${ }^{1,2}$

Muscle fatigue can be viewed as either central and/or peripheral in origin. ${ }^{3}$ Central fatigue refers

Abbreviations: RM, repetition maximum; ANOVA, analysis of variance; EMG, Electromyographic; HIRC, High intensity resistance circuit; HR, heart rate; ICC, intra-class correlation coefficient; $\mathrm{M}_{\max }$, maximum muscle compound action potential; MVC, maximal voluntary contraction; $Q_{t w}$, resting twitch amplitude; $\mathrm{Pi}$, inorganic phosphate; RMS, root mean square; RPE, rating of perceived exertion; TST, traditional strength training; VA, voluntary activation; VL, vastus lateralis

Key words: fatigue; metabolic response; perception of effort; resistance training; neuromuscular function; twitch interpolation technique

Funding: This study was supported by Catholic University of Murcia (Ref.: PMAFI/23/14).

Conflicts of Interest: All authors have no conflict of interest and no further financial disclosure to make.

Correspondence to: G. Márquez, Campus de los Jerónimos s/n. 30107Guadalupe, Facultad del Deporte, Murcia. Spain; e-mail: gmarquez@ ucam.edu

(C) 2016 Wiley Periodicals, Inc.

Published online 00 Month 2016 in Wiley Online Library (wileyonlinelibrary. com). DOI 10.1002/mus.25460

Fatigue in Heavy Circuit Training to a reduction in voluntary activation during exercise, while peripheral fatigue indicates impairment in force-generating capacities at or distal to the neuromuscular junction, including metabolic and biochemical changes within the muscle. ${ }^{3}$ The relative magnitude of central and peripheral fatigue depends on many factors, such as the duration, intensity, and type of exercise. In this regard, several studies have explored the fatigue responses elicited by different types of exercise and loads. Recent studies have shown that high intensity whole body exercise exacerbates both central and peripheral fatigue., ${ }^{4,5}$ Heavy resistance training loads (2-6 repetition maximum, RM) have been shown to induce central fatigue, ${ }^{6,7}$ while lower loads (10-20 RM) are assumed to elicit hypertrophy by means of a peripheral response in the muscle. $^{8}$

However, no study has been conducted to investigate central and peripheral fatigue induced by a modality of resistance training known as circuit training. Circuit training is customarily defined by the combination of several exercises, $12-15$ repetitions per set, light loads $(\sim 40 \%$ of 1 $\mathrm{RM})$, and short rest periods (15-30 s) between exercises. ${ }^{9}$ Circuit weight training is very effective at increasing maximum oxygen consumption, maximum pulmonary ventilation, local muscle endurance, functional capacity, and lean body mass, while reducing body fat. ${ }^{10-12}$ Along this line, a new version of circuit weight training using higher loads (i.e., 6RM) and shorter rest periods (i.e., 35 s) has been proposed recently. High intensity resistance circuit (HIRC) training has been shown to elicit even greater benefits than traditional circuit training. ${ }^{13,14}$ HIRC training, therefore, seems to be a time-efficient model of resistance training that uses reduced rest periods to increase the metabolic, ${ }^{15}$ hormonal, ${ }^{16,17}$ and cardiovascular responses. ${ }^{18}$

Comparing the effects of a single training session in young adults, Alcaraz et al. ${ }^{19}$ found that, with the same loading (i.e., 6RM), strength, power, and total workload were similar between an HIRC and a traditional strength training (TST) session. However, the cardiorespiratory response measured 


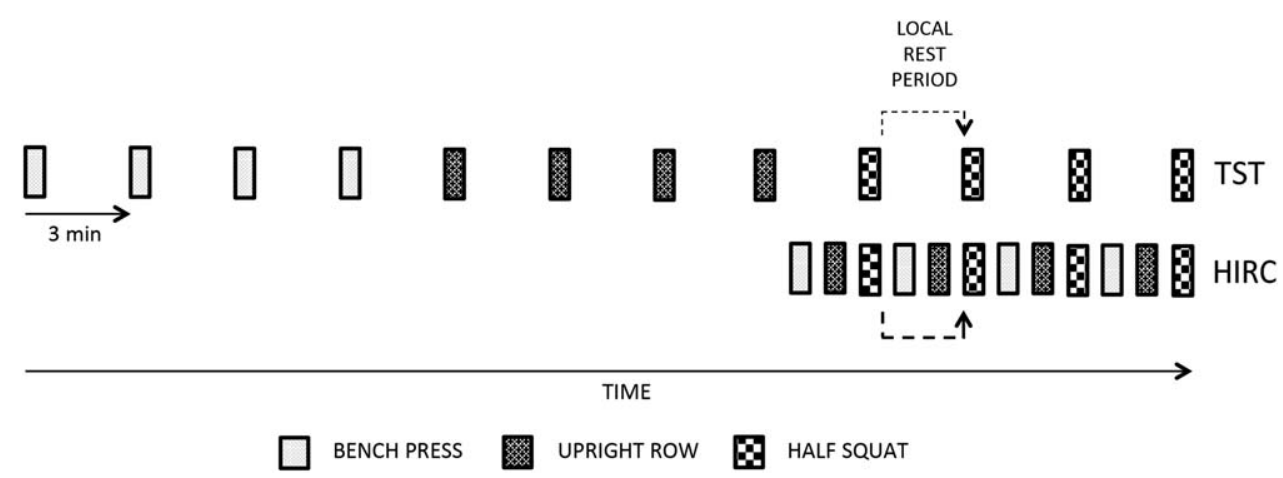

FIGURE 1. Set configuration of both TST and HIRC sessions. Here, only the first block is illustrated, however, the subjects were asked to perform 2 blocks of 4 sets separated by $5 \mathrm{~min}$. Note that the inter-set times, i.e., the local rest periods for each muscle, were the same in TST and HIRC, however, the breaks between exercises and the total training duration differed.

by heart rate (HR) was greater with HIRC $(71 \%$ maximum HR) than with TST training $(62 \%$ maximum HR). So far, the neuromuscular demands associated with HIRC training have not been addressed, and thus, it is unknown whether HIRC training differently affects the peripheral and central fatigue mechanisms. Therefore, the aim of this study was to compare the acute effects on neuromuscular function of HIRC and TST training sessions. In addition, metabolic responses and rating of perceived exertion were evaluated. Although the loads and rest periods for each trained (synergistic) muscle group were matched for both TST and HIRC (180 s), we hypothesized that HIRC produces a more intense acute metabolic response due to its lower inter-exercise time (HIRC: $35 \mathrm{~s}$; TST: $155 \mathrm{~s}$ ). This increase in metabolic stress in the HIRC compared with the TST session is assumed to lead to higher peripheral fatigue. However, it may also influence central mechanisms differently, as it has been demonstrated that afferents III and IV, which sense metabolite accumulation such as lactate, $\mathrm{H}^{+}$, and ATP, ${ }^{20}$ have been reported to partially inhibit corticospinal drive. ${ }^{21}$

\section{MATERIALS AND METHODS}

Subjects. Twelve healthy men (age: $21 \pm 0.8$ years; height: $178 \pm 5 \mathrm{~cm}$; weight: $78.2 \pm 7.3 \mathrm{~kg}$ ) were recruited for this study. The subjects were students of the Faculty of Sport Sciences in the Catholic University of Murcia, and all performed $3.5 \pm 0.8 \mathrm{~h}$ per week of vigorous physical activity including at least 1 strength training session per week, with $\geq 3$ years of experience. All subjects gave written consent to the experimental procedure. The experimental procedures were performed in accordance with the Declaration of Helsinki and were approved by the local ethics committee.

Experimental Protocol. Each subject was involved in 1 familiarization and 2 experimental sessions with a break of 1 week between sessions. The familiarization was performed 1 week before the first experimental session. This session was conducted to acquaint the subjects with the different exercises (bench press, upright row, and back squat), and to establish the individual 6RM load (the load that induced failure after 6 repetitions) for each exercise. During this session, subjects were also required to perform 2 isometric maximal voluntary contractions (MVCs) with and without electrical stimulation. These data were then analyzed, and the intra-class correlation coefficients (ICCs) were computed for maximal voluntary contraction, voluntary activation (VA), and resting potentiated twitch amplitude $\left(Q_{\mathrm{tw}}\right)$ to test reliability of this procedure (ICCs were higher than 0.80 in all the variables measured).

After the familiarization session, subjects performed 2 different strength-training sessions (HIRC and TST) in a counterbalanced order 1 week apart. Each session consisted of 8 sets of 6 maximum repetitions (6RM) of the 3 different exercises. The work load (set and session volume) and inter-set periods (180 s) were the same in both training sessions. Thus, the time between the different exercises was $35 \mathrm{~s}$ during HIRC, whereas during TST, subjects rested for $155 \mathrm{~s}$. The reason for this difference was based on the fact that exercises in the HIRC training session were alternated, whereas they were not during TST (Fig. 1).

Importantly, in every training session, half squat was the last exercise before the postmeasurements started. Thus, neuromuscular function of knee extensor muscles and metabolic responses were tested before and 1, 4, 7, and $10 \mathrm{~min}$ after the respective training protocol.

Procedures. Motor nerve stimulation. A single supramaximal electrical pulse (1 ms) was applied to the femoral nerve of the right leg by means of adhesive electrodes ( $4 \mathrm{~cm}$ diameter) using a Digitimer DS7AH (Welwyn Garden City, UK). The 
cathode was placed over the femoral triangle, and the anode was positioned midway between the greater trochanter and the iliac crest. Both electrodes were made of carbonized rubber, coated with an electroconductive gel. Before placing the cathode, a small electrode $(2 \times 2 \mathrm{~cm})$ was placed on the femoral triangle to determine the best position for femoral nerve stimulation. The intensity of the electrical pulse was set to $120 \%$ of that required to elicit maximum quadriceps twitch amplitude $\left(\mathrm{Q}_{\mathrm{tw}}\right)$ and muscle compound action potential $\left(\mathrm{M}_{\max }\right)$. This intensity was maintained constant throughout the protocol. The final stimulation intensity was $204 \pm 87 \mathrm{~mA}$.

Neuromuscular function test. To test central and peripheral fatigue, the subjects were placed in a seated position on a Biodex System $3^{\circledR}$ dynamometer and were securely strapped into the test chair. The chair had a long backrest to provide full back support. The hip and knee were fixed at $90^{\circ}$ of flexion.

The twitch interpolation technique was used to assess neuromuscular function of knee extensor muscles before the beginning of each training session (HIRC and TST) and after 1, 4, 7, and $10 \mathrm{~min}$ posttraining. According to Herbert and Gandevia, ${ }^{22}$ electrical motor nerve stimulation was delivered during a 2- to 3-s steady torque plateau of the MVC. Thereafter, 3 additional single electrical stimulations were applied at rest with a 2-s interval between them (the mean of the 3 resting potentiated twitches was taken into account for further analysis). For a reliable measurement of the MVC, methodological recommendations from Gandevia ${ }^{3}$ were taken into consideration.

$E M G$ and torque recordings. Electromyographic (EMG) activity was recorded from the vastus lateralis (VL) muscle of the right leg, using pregelled surface $\mathrm{Ag}-\mathrm{AgCl}$ electrodes with an inter-electrode distance of $20 \mathrm{~mm}$. The electrode placement was in accordance with SENIAM recommendations. ${ }^{23}$ Before application of the electrodes, the skin surface was shaved, abraded, and cleaned with alcohol. To reduce movement artifact, the electrodes and the unit were taped firmly. The electrodes were kept in place throughout the experimental session and connected to a wireless data acquisition system (Kine Pro $^{\circledR}$ ). EMG activity was recorded at a $1,600 \mathrm{~Hz}$ sampling rate. All signals were amplified and filtered with a bandwidth of 16-500 Hz, where each channel has an input impedance $>100 \mathrm{MOhm}$, common mode rejection ratio $>100 \mathrm{~dB}$, and a gain of 1,000 . All data were stored on a PC for off-line processing and analysis. Voluntary and evoked torque were measured using the isokinetic Biodex System $3^{\circledR}$ dynamometer with a sampling frequency of $100 \mathrm{~Hz}$. Both torque and
EMG signals were synchronized using an external digital trigger.

Blood sampling and rating of perceived exertion (RPE). Blood lactate concentration was determined using a Lactate Pro Analyzer (Arkray; Japan) from capillary blood samples drawn from the hyperemic ear lobe $1 \mathrm{~min}$ before the beginning of each resistance training bout and after $1,4,7$, and $10 \mathrm{~min}$ posttraining bout (HIRC vs. TST).

According to the Borg Rating of Perceived Exertion (RPE) scale (6 to 20 points) for perceived exertion, ${ }^{24}$ the subjects reported their RPE for each voluntary contraction performed $1 \mathrm{~min}$ before and after 1, 4, 7, and 10 min post training (HIRC and TST). They were instructed to choose the number from the 6-20 scale that best described the level of exertion and fatigue $(6=$ not exerted or fatigued at all; $20=$ maximally exerted and fatigued).

Training protocols. The loads corresponding to $6 \mathrm{RM}$ were $79.3 \pm 5.8,40.4 \pm 1.9$, and $106.8 \pm 8.9 \mathrm{~kg}$ for the bench press, upright row, and back squat, respectively. Figure 1 shows the set configuration for TST and HIRC.

TST. After a general warm-up, the subjects performed a specific warm-up consisting of 2 sets of 3 exercises (bench press, upright row, and back squat) using the following sequence: 12 repetitions at $50 \%$ of $6 \mathrm{RM}$ and after 1-min rest, 10 repetitions at $75 \%$ of $6 \mathrm{RM}$ followed by a 2-min rest. After the specific warm-up, subjects performed the TST protocol, which consisted of 8 sets of 6RM of each exercise distributed in 2 blocks of 4 sets separated by $5 \mathrm{~min}$. The inter-set period was $180 \mathrm{~s}$. Thus, the rest between the 3 different exercises was $155 \mathrm{~s}$. The eccentric phase of each exercise was performed over approximately $3 \mathrm{~s}$ (controlled by a metronome app for iPhone ${ }^{\circledR}$ ), whereas the concentric phase was performed at maximum velocity. The total training time in the TST protocol was $\sim 77 \mathrm{~min}$.

HIRC training. Training performed in the HIRC session differed from TST only in the rest interval between the exercises and the sequencing of exercises. While subjects in the TST session performed the exercises one after the other with an inter-set period of $180 \mathrm{~s}$, subjects in the HIRC protocol executed the training in a circuit manner. Thus, bench press, upright row, and back squat exercises were performed in a successive sequence with a $35 \mathrm{~s}$ recovery period between exercises. Importantly, the local rest period (155 s) for each specific muscle group used for the 3 exercises was exactly the same in HIRC and TST. Furthermore, the warm-up, the intensity (6RM), and the volume (8 sets) were identical in HIRC and TST. As in the TST protocol, the training session was distributed 
in 2 blocks of 4 sets separated by $5 \mathrm{~min}$. However, the total training time in the HIRC session was only $\sim 29 \mathrm{~min}$.

All the exercises performed during the experimental sessions were conducted on a modified Smith machine (Technogym ${ }^{\circledR}$, Italy). To control the correct execution of the half squat movement, participants were instructed to reach a knee angle of $90^{\circ}$. Moreover, during both TST and HIRC sessions, subjects were supervised by an experienced weight lifter. The duration of the rest intervals was strictly controlled and standardized.

To prevent the adverse effect of dehydration on neuromuscular performance, ${ }^{25}$ subjects were asked to drink $200 \mathrm{ml}$ of water during the 5-min rest period (after the fourth set) and just after the last set (eighth) of both training sessions.

Data analysis. The amplitude of the superimposed twitch was obtained by subtracting the torque at the onset of each twitch from the peak torque. This was done at rest and during the MVC conditions. VA was obtained for trials with $100 \%$ MVC and was calculated according to the following equation:

$\mathrm{VA}(\%)=[1-($ superimposed twitch/resting twitch $)] \times 100$

During contractions in which electrical stimuli were delivered, the $M_{\max }$ amplitude (peak to peak) and root mean square (RMS) EMG (between 1,005 and $5 \mathrm{~ms}$ before stimulation) were measured for the VL muscle. According to Girard et al. ${ }^{26}$ raw RMS_VL data were normalized to the superimposed M-wave as an index of neural drive (i.e. RMS_VL / $M_{\max }$ ratio).

Statistical Analysis. All data were normally distributed and met the assumptions for linear statistical analysis. Therefore, 2-way analyses of variance (ANOVAs) of repeated measures were performed with session (HIRC and TST) and time (Pre, Post_1', Post_4', Post_7', and Post_10') as factors. The ANOVAs were performed for the following variables: MVC, potentiated resting twitch amplitude $\left(Q_{\mathrm{tw}}\right.$ in $\left.\mathrm{N} \cdot \mathrm{m}^{-1}\right)$, VA (in $\left.\%\right)$, blood lactate concentration (in $\mathrm{mmol} \cdot \mathrm{l}^{-1}$ ), rating of perceived exertion, $M_{\max }$ (in $\mathrm{mV}$ ), RMS_VL (in $\mu \mathrm{V}$ ), and RMS_VL/ $\mathrm{M}_{\max }\left(\right.$ in $\left.\mu \mathrm{V} \cdot \mathrm{mV}^{-1}\right)$. Post hoc analysis was performed using paired comparisons with Bonferroni correction. Partial Eta Square $\left(\eta_{\mathrm{P}}^{2}\right)$ and Cohen $d$ were calculated as effect sizes. Pearson correlation coefficients were calculated to determine the relationship between the percent increase in blood lactate concentration and percent decrease of MVC, VA, and $Q_{\mathrm{tw}}$ after both the TST and HIRC sessions. SPSS 20.0 software (SPSS, Chicago, Illinois) was used for statistical analysis. The sample size was calculated using the G POWER software

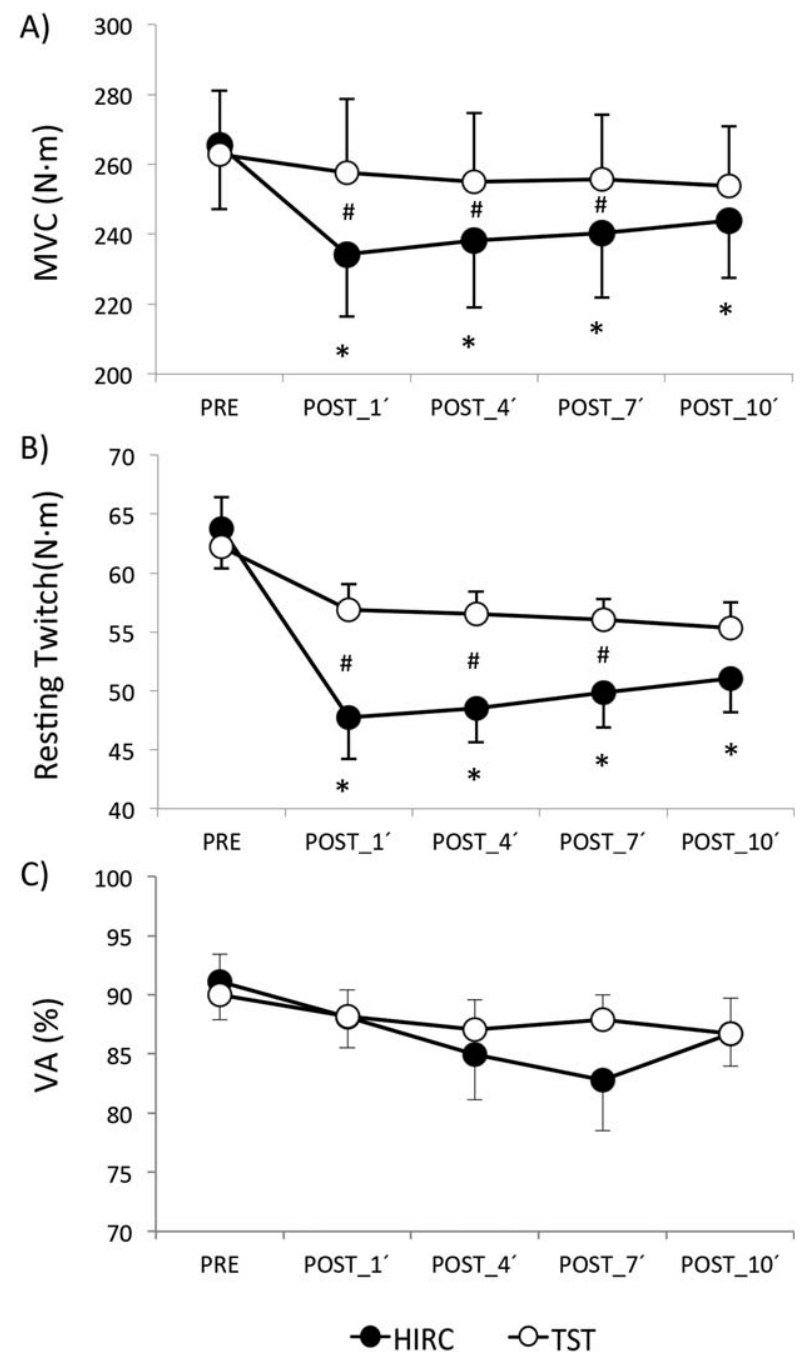

FIGURE 2. Differences in MVC (A), Resting Twitch Amplitude (B), and Voluntary Activation (C) between the HIRC and TST sessions. Values are shown as mean $\pm \mathrm{SE}$. *differences related to baseline after the HIRC session. \#differences between HIRC and TST sessions.

(version 3.0.1) which determined that a sample of $n=12$ subjects would provide a statistical power of $>0.80$ in all variables. The retrospective statistical power provided by SPSS after analysis was $>0.9$ in the MVC, $Q_{t w}, V A$, lactate, and $R P E$ variables in which a significant time-effect was observed. The retrospective statistical power for the variables which showed significant time vs. training interactions in the results $\left(\mathrm{MVC}, \mathrm{Q}_{\mathrm{tw}}\right.$, lactate, and $\left.\mathrm{RPE}\right)$ were $>0.9$, with the exception of the MVC, for which the statistical power was 0.7. Statistical significance was set at $P \leq 0.05$. Data are presented as means $\pm \mathrm{SD}$ in the text and tables, and means $\pm \mathrm{SE}$ in the figures.

\section{RESULTS}

MvC, Resting Twitch Amplitude, and Voluntary Activation. The results of the MVC are shown in Figure 2A. There was a main effect for TIME 
Table 1. Mean $( \pm S D)$ of the lactate and RPE values before and after HIRC and TST

\begin{tabular}{lcccccc}
\hline Variables & Training & PRE & POST_1 & POST_4 & POST_7' & POST_10' \\
\hline La $\left(\right.$ mmol. I $^{-1}$ ) & HIRC & $1.6 \pm 0.6$ & $11.6 \pm 2.2^{*, \dagger}$ & $10.8 \pm 2.4^{*, \dagger}$ & $9.8 \pm 2.4^{*, \dagger}$ & $9.0 \pm 2.6^{*, \dagger}$ \\
& TST & $1.6 \pm 0.6$ & $4.7 \pm 1.6^{\ddagger}$ & $3.6 \pm 1.4^{\ddagger}$ & $3.2 \pm 1.3^{\ddagger}$ & $2.6 \pm 0.9^{\ddagger}$ \\
RPE (a.u.) & HIRC & $8.2 \pm 1.3$ & $17.5 \pm 2.7^{*, \dagger}$ & $16.3 \pm 2.6^{*, \dagger}$ & $15.5 \pm 2.7^{*, \dagger}$ & $15.1 \pm 3.1^{*, \dagger}$ \\
& TST & $8.7 \pm 1.8$ & $12.5 \pm 2.2^{\ddagger}$ & $12.0 \pm 1.9^{\ddagger}$ & $11.8 \pm 2.0^{\ddagger}$ & $11.3 \pm 2.3^{\ddagger}$ \\
\hline
\end{tabular}

La, lactate; RPE, rating of perceived exertion; HIRC, high intensity resistance circuit; TST, traditional strength training.

*Differences related to baseline (PRE) after HIRC.

${ }^{\dagger}$ Differences between HIRC and TST sessions.

${ }^{\ddagger}$ Differences related to baseline (PRE) after TST.

$\left(\mathrm{F}=5.77 ; P=0.001 ; \eta_{\mathrm{P}}^{2}=0.344\right)$ and a significant TRAINING x TIME interaction $(\mathrm{F}=2.69 ; P=0.049$; $\left.\eta_{\mathrm{P}}^{2}=0.191\right)$. Post hoc analysis showed a significant reduction in the $\mathrm{MVC}$ values at Post_1' $(-11.6 \% \pm$ $12.1 \%)$, Post_4' $(-10.7 \% \pm 8.9 \%)$, Post_7' $(-9.8 \% \pm$ $8.5 \%)$, and Post_10' $(-7.8 \% \pm 7.5 \%)$ test blocks compared with pretest for HIRC $(P<0.01$ and $\mathrm{d}>0.40$ for all paired comparisons). No significant torque decrease was found after TST. Paired comparisons also revealed significant differences between both training protocols at Post_1' $\quad(\mathrm{t}=23.34$, $P=0.045 ; \mathrm{d}=0.36)$, Post_2 $2^{\prime}(\mathrm{t}=16.90, P=0.036$; $\mathrm{d}=0.26)$, and Post_ $7^{\prime}(\mathrm{t}=15.45 ; P=0.049 ; \mathrm{d}=0.25)$.

Regarding the $Q_{\mathrm{w}}$ amplitudes, ANOVA of repeated measures revealed a main effect for TIME $\left(\mathrm{F}=28.23 ; P<0.001 ; \eta_{\mathrm{P}}^{2}=0.720\right)$ and a significant TRAINING $\quad \mathrm{x}$ TIME interaction $(\mathrm{F}=10.76$; $\left.P<0.001 ; \eta_{\mathrm{P}}^{2}=0.494\right)$. Post hoc analysis showed a significant reduction in the $Q_{\mathrm{tw}}$ values at Post_1 ${ }^{\prime}$ $(-23.9 \% \pm 16.1 \%), \quad$ Post_4 $4^{\prime} \quad(-22.6 \% \pm 12.8 \%)$, Post_7' $(-20.4 \% \pm 13.3 \%)$, and Post_10' $(-18.9 \% \pm$ $12.5 \%)$ test blocks compared with pretest for HIRC $(P<0.01$ and $\mathrm{d}>1.40$ for all paired comparisons; Fig. 2B). No significant changes were found after TST. In addition, paired comparisons revealed significant differences between both training protocols at Post_1' $1^{\prime}(\mathrm{t}=9.17 ; \quad P=0.025$; $\mathrm{d}=0.97)$, Post_2' $(\mathrm{t}=8.01, P=0.016 ; \mathrm{d}=1.04)$, and Post_7' $(\mathrm{t}=6.18 ; P=0.040 ; \mathrm{d}=0.82)$ (Fig. 2B).

The ANOVA of repeated measures of VA showed a main effect for the factor TIME $\left(\mathrm{F}=4.71 ; P=0.003 ; \eta_{\mathrm{P}}^{2}=0.300\right)$. There was no significant TRAINING X TIME difference $(\mathrm{F}=1.809$; $\left.P=0.144 ; \eta_{\mathrm{P}}^{2}=0.141\right)$, although visual inspection of the data may indicate differences between groups at Post_4' and Post_7' (Fig. 2C). However, not even uncorrected $t$-tests at Post_4 $4^{\prime}(\mathrm{P}=0.382$; $\mathrm{d}=0.45), \quad$ and $\quad$ Post_7 $\quad(\mathrm{P}=0.180 ; \quad \mathrm{d}=0.29)$ revealed significant differences between HIRC and TST, indicating comparable decreases of voluntary activation after both kinds of training sessions.

Blood Lactate Concentration and RPE. Regarding blood lactate concentration, ANOVA of repeated measures showed a main effect for TRAINING
$\left(\mathrm{F}=145.89 ; \quad P<0.001 ; \quad \eta_{\mathrm{P}}^{2}=0.930\right), \quad$ TIME $\left(\mathrm{F}=155.26 ; \quad P<0.001 ; \eta_{\mathrm{P}}^{2}=0.934\right)$, and a significant TRAINING $\times$ TIME interaction $(\mathrm{F}=78.49$; $\left.P<0.001 ; \eta_{\mathrm{P}}^{2}=0.877\right)$. Results from lactate concentration are displayed in Table 1. It should be noticed that, after both training protocols, lactate concentration values in all the posttraining test blocks (Post_1', Post_4', Post_7', and Post_10') were higher than before the beginning of each training session $(P<0.005$ for all comparisons). Post hoc analysis also revealed higher metabolic stress (lactate production) after the HIRC training compared with TST training at Post_1' $(\mathrm{t}=6.9$; $P<0.001 ; \quad \mathrm{d}=4.43), \quad$ Post_4 $4^{\prime} \quad(\mathrm{t}=7.2 ; \quad P<0.001$; $\mathrm{d}=4.43)$, Post_7' $(\mathrm{t}=6.6 ; \bar{P}<0.001 ; \mathrm{d}=3.79)$, and Post_10' (t $=6 . \overline{4} ; P<0.001 ; \mathrm{d}=4.95)$ (see Table 1$)$.

Results from RPE are shown in Table 1. ANOVA of repeated measures showed a main effect for TRAINING $\left(\mathrm{F}=69.05 ; P<0.001 ; \eta_{\mathrm{P}}^{2}=\right.$ $0.863)$, TIME $\left(\mathrm{F}=65.47 ; \quad P<0.001 ; \quad \eta_{\mathrm{P}}^{2}=0.856\right)$, and a significant TRAINING $\mathrm{x}$ TIME interaction $\left(\mathrm{F}=30.26 ; P<0.001 ; \eta_{\mathrm{P}}^{2}=0.733\right)$. RPE values were significantly increased after both training sessions in comparison to the pretest $(P<0.005$ for all comparisons). Post hoc analysis revealed higher subjective ratings of effort after HIRC compared with TST at Post_1', Post_4', Post_7', and Post_10' $(P<0.001$ and $\mathrm{d}>1.80$ for all comparisons; Table 1$)$.

There were no significant correlations between the percent increases in blood lactate and the percent reductions in the resting twitch amplitude, VA, or MVC.

VL EMG and $\mathbf{M}_{\max }$. There were no differences in the peak-to-peak amplitude of $\mathbf{M}_{\max }$ before and after the HIRC and TST sessions. These data indicate that membrane excitability of the motor nerve remained unchanged. ${ }^{27}$ In addition, VL EMG activity (both RMS_VL and RMS_VL/M $M_{\max }$ ) did not change in response to the training session (see Table 2).

\section{DISCUSSION}

In this study, we investigated peripheral and central fatigue of knee extensor muscles in 
Table 2. Mean $( \pm S D)$ of the EMG recordings during MVCs performed before and after HIRC and TST

\begin{tabular}{lcccccr}
\hline Variables & Training & PRE & POST_1' $^{\prime}$ & POST_4' $^{\prime}$ & POST_7' $^{\prime}$ & POST_10' $^{\prime}$ \\
\hline RMS_VL $(\mu \mathrm{V})$ & HIRC & $215 \pm 102$ & $198 \pm 82$ & $205 \pm 87$ & $204 \pm 93$ & $205 \pm 68$ \\
& TST & $231 \pm 80$ & $219 \pm 74$ & $220 \pm 88$ & $205 \pm 76$ & $223 \pm 86$ \\
$M_{\max }(\mathrm{mV})$ & HIRC & $5.3 \pm 1.8$ & $4.9 \pm 1.6$ & $4.9 \pm 1.4$ & $4.9 \pm 1.4$ & $4.9 \pm 1.4$ \\
RMS_VL/M $_{\max }\left(\mu \mathrm{V} \cdot \mathrm{mV}^{-1}\right)$ & TST & $5.2 \pm 1.8$ & $4.8 \pm 1.5$ & $4.8 \pm 1.7$ & $4.8 \pm 1.7$ & $4.8 \pm 1.7$ \\
& HIRC & $40.6 \pm 14$ & $40.5 \pm 17$ & $41.8 \pm 17$ & $41.6 \pm 17$ & $41.8 \pm 14$ \\
& TST & $44.5 \pm 17$ & $45.6 \pm 17$ & $45.8 \pm 17$ & $42.7 \pm 14$ & $46.5 \pm 17$ \\
\hline
\end{tabular}

RMS VL, root mean square of the vastus lateralis muscle; $M_{\max }$ compound muscle action potential; RMS VL/M max ratio between the root mean square of the vastus lateralis and the compound muscle action potential; HIRC, high intensity resistance circuit; TST, traditional strength training.

response to HIRC and TST. HIRG induced a reduction in the maximal isometric voluntary contraction associated with a decrease in the resting twitch amplitude and high metabolic responses. As VA was reduced after both training sessions, we argue that mechanisms at the muscular level are primarily responsible for the differences between HIRC and TST.

Voluntary Activation Indicating Central Fatigue. Analysis of VA indicated comparable impairment in maximal voluntary neural drive to knee extensors after HIRC and TST. These results are in line with a previous study, in which similar training loads were shown to induce central fatigue. ${ }^{7}$ It has been also demonstrated that whole body exercises such as repeated high intensity cycling or running sprints produce similar amounts of central fatigue. ${ }^{4,5}$

Neither the RMS_VL nor the RMS_VL/M max were affected differently by HIRC and TST. However, HIRC but not TST led to reduced MVC of knee extensor muscles that lasted for at least $10 \mathrm{~min}$ after the end of exercise. Recent studies have also shown a mismatch between changes in VA level and $\mathrm{RMS} / \mathrm{M}_{\max }$ ratio $^{4,28}$ indicating that the EMG signal is not a reliable index of central motor drive. ${ }^{28}$ As central fatigue seems not to be responsible for this difference between HIRC and TST, peripheral mechanisms are more likely.

Peripheral Fatigue, Blood Lactate Concentration, and RPE. A decrease in the resting twitch amplitude after HIRC suggests that peripheral mechanisms contributed to the reduction of MVC. Thus, it is likely that the contractile properties of muscle fibers and excitation-contraction coupling became compromised after the HIRC session as a result of peripheral fatigue. ${ }^{29,30}$ The reduction in resting twitch amplitude in response to motor nerve stimulation could result from several factors such as the accumulation of $\mathrm{H}^{+}$and inorganic phosphate (Pi) that leads to a reduction in $\mathrm{Ca}^{2+}$ sensitivity and a decrease in the number of strong binding cross bridges. $^{31,32}$
The most interesting question is why only the HIRC session induced peripheral fatigue in knee extensor muscles when the total time under tension and local resting periods for each muscle group were identical between HIRC and TST. A difference between HIRC and TST was the level of accumulated lactate: HIRC induced lactate concentrations $\left(11.6 \pm 2.2 \mathrm{mMol} \cdot \mathrm{l}^{-1}\right)$ comparable to those reported after a Wingate test ${ }^{33}$ and after repeated maximal intensity-sprints on a cycle ergometer. ${ }^{5}$ In contrast, TST resulted in much lower lactate concentrations $\left(4.7 \pm 1.6 \mathrm{mMol} \cdot \mathrm{l}^{-1}\right)$. Lactate accumulation seems to be determined not primarily by the duration of breaks between local muscle groups but rather depends on the breaks between the different exercises.

In favor of this assumption, previous studies have shown that exhaustive anaerobic exercises by the arms (or legs) might decrease the performance of the nonexercised legs (or arms) in subsequent anaerobic efforts. ${ }^{34,35}$ The authors suggested that lactate elevation might cause an increased uptake in $\mathrm{La}^{-}$and $\mathrm{H}^{+}$by both the active and inactive muscles, leading to an overall performance decrement. Although it is known by now that lactate per $s e$ does not directly cause muscle fatigue, a rise in other metabolic byproducts such as $\mathrm{Pi}$ could play a major role in muscle fatigue during high intensity exercise. $^{36}$ High Pi levels can impair contractile function by several mechanisms such as reducing myofibrillar $\mathrm{Ca}^{2+}$ sensitivity or decreasing the amount of $\mathrm{Ca}^{2+}$ available for release. ${ }^{37}$

Thus, a depletion and inhibition of the phosphagen and glycolytic energy systems as a result of a rise in metabolic byproducts during exercise ${ }^{38}$ may affect muscle function in the nonexercised arm or leg. ${ }^{39,40}$ Therefore, the high blood lactate concentration levels associated with HIRC probably indicate a rise in metabolic byproducts in general, which in turn led to a decline in muscle force production. In addition, the decrease in resting twitch amplitudes after HIRC indicates impaired excitation-contraction coupling as a result of metabolite accumulation during the exercise. ${ }^{32,41}$ However, 
this assumption should be considered with caution, as the role of $\mathrm{pH}$ in the reduction of $\mathrm{MVC}, \mathrm{VA}$, and $Q_{t w}$ has recently been questioned. ${ }^{42}$ In addition, we did not find correlations between lactate and any of the neuromuscular parameters studied, so further research is needed to address this question.

Similar to the results obtained for blood lactate concentration, RPE was higher after HIRC compared with TST. This confirms previous studies that show high correlations between the lactate concentration and the rating of self-perceived exertion. $^{43}$ It has been recently argued that afferent feedback from locomotor muscles plays a critical role in determining perception of effort. ${ }^{44}$

In conclusion, our results show that HIRC induced a reduction in MVC, larger metabolic responses and higher ratings of perceived exertion in comparison with TST. The reduction in MVC after HIRC was mainly caused by peripheral fatigue, as indicated by reduced resting twitch amplitudes. These findings suggest that HIRC training sessions have a more intensive acute effect on muscular and metabolic responses than traditional resistance training sessions.

The authors are grateful to the subjects who contributed to this study.

\section{REFERENCES}

1. Schott J, McCully K, Rutherford O. The role of metabolites in strength training. Eur J Appl Physiol Occup Physiol 1995;71:337-341.

2. Rooney KJ, Herbert RD, Balnave RJ. Fatigue contributes to the strength training stimulus. Med Sci Sports Exerc 1994;26:1160-1164.

3. Gandevia SC. Spinal and supraspinal factors in human muscle fatigue. Physiol Rev 2001;81:1725-1789.

4. Goodall S, Charlton K, Howatson G, Thomas K. Neuromuscular fatigability during repeated-sprint exercise in male athletes. Med Sci Sports Exerc 2015;47:528-536.

5. Pearcey GE, Murphy JR, Behm DG, Hay DC, Power KE, Button DC. Neuromuscular fatigue of the knee extensors during repeated maximal intensity intermittent-sprints on a cycle ergometer. Muscle Nerve 2015;51:569-579.

6. Kraemer WJ, Ratamess NA. Fundamentals of resistance training: progression and exercise prescription. Med Sci Sports Exerc 2004;36: $674-688$.

7. Walker S, Davis L, Avela J, Häkkinen K. Neuromuscular fatigue during dynamic maximal strength and hypertrophic resistance loadings. J Electromyogr Kinesiol 2012;22:356-362

8. Behm DG, Reardon G, Fitzgerald J, Drinkwater E. The effect of 5, 10 , and 20 repetition maximums on the recovery of voluntary and evoked contractile properties. J Strength Cond Res 2002;16:209-218.

9. Tesch PA 1992. Training for bodybuilding. In: Komi PV, editor. Strength and power in sport. Oxford: Blackwell Scientific Publications. p 370-381.

10. Camargo MD, Stein R, Ribeiro JP, Schvartzman PR, Rizzatti MO, Schaan BD. Circuit weight training and cardiac morphology: a trial with magnetic resonance imaging. Br J Sports Med 2008;42:141-145.

11. Harber MP, Fry AC, Rubin MR, Smith JC, Weiss LW. Skeletal muscle and hormonal adaptations to circuit weight training in untrained men. Scand J Med Sci Sports 2004;14:176-185.

12. Monteiro WD, Simao R, Polito MD, Santana CA, Chaves RB, Bezerra $\mathrm{E}$, et al. Influence of strength training on adult women's flexibility. J Strength Cond Res 2008;22:672-677.

13. Paoli A, Pacelli F, Bargossi AM, Marcolin G, Guzzinati S, Neri M, et al. Effects of three distinct protocols of fitness training on body composition, strength and blood lactate. J Sports Med Phys Fitness 2010;50:43-51.

14. Paoli A, Pacelli OF, Moro T, Marcolin G, Neri M, Battaglia G, et al. Effects of high-intensity circuit training, low-intensity circuit training and endurance training on blood pressure and lipoproteins in middle-aged overweight men. Lipids Health Dis 2013;12:131.
15. Kraemer WJ, Noble B, Clark M, Culver B. Physiologic responses to heavy-resistance exercise with very short rest periods. Int J Sports Med 1987;8:247-252.

16. Kraemer WJ, Gordon S, Fleck S, Marchitelli L, Mello R, Dziados J, et al. Endogenous anabolic hormonal and growth factor responses to heavy resistance exercise in males and females. Int J Sports Med 1991;12:228-235.

17. Kraemer WJ, Fleck SJ, Dziados JE, Harman EA, Marchitelli LJ Gordon SE, et al. Changes in hormonal concentrations after different heavy-resistance exercise protocols in women. J Appl Physiol 1993;75:594-604.

18. Fleck SJ. Cardiovascular adaptations to resistance training. Med Sci Sports Exerc 1988;20(Suppl):S146-S151.

19. Alcaraz PE, Sanchez-Lorente J, Blazevich AJ. Physical performance and cardiovascular responses to an acute bout of heavy resistance circuit training versus traditional strength training. J Strength Cond Res 2008;22:667-671

20. Light AR, Hughen RW, Zhang J, Rainier J, Liu Z, Lee J. Dorsal root ganglion neurons innervating skeletal muscle respond to physiological combinations of protons, ATP, and lactate mediated by ASIC, P2X, and TRPV1. J Neurophysiol 2008;100:1184-1201.

21. Amann M, Dempsey JA. Locomotor muscle fatigue modifies central motor drive in healthy humans and imposes a limitation to exercise performance. J Physiol 2008;86:161-173.

22. Herbert RD, Gandevia SC. Twitch interpolation in human muscles: mechanisms and implications for measurement of voluntary activation. J Neurophysiol 1999;82:2271-2283.

23. Hermens HJ, Freriks B, Disselhorst-Klug C, Rau G. Development of recommendations for SEMG sensors and sensor placement procedures. J Electromyogr Kinesiol 2000;10:361-374.

24. Borg GA. Psychophysical bases of perceived exertion. Med Sci Sports Exerc 1982;14:377-381.

25. Vallier JM, Grego F, Basset F, Lepers R, Bernard T, Brisswalter J. Effect of fluid ingestion on neuromuscular function during prolonged cycling exercise. Br J Sports Med 2005;39:e17.

26. Girard O, Bishop DJ, Racinais S. Neuromuscular adjustments of the quadriceps muscle after repeated cycling sprints. PloS One 2013;8: e61793.

27. Fowles JR, Green HJ, Tupling R, O'Brien S, Roy BD. Human neuromuscular fatigue is associated with altered $\mathrm{Na}^{+}-\mathrm{K}^{+}$-ATPase activity following isometric exercise. J Physiol 2002;92:1585-1593.

28. Pageaux B, Angius L, Hopker JG, Lepers R, Marcora SM. Central alterations of neuromuscular function and feedback from group IIIIV muscle afferents following exhaustive high-intensity one-leg dynamic exercise. Am J Physiol Regul Integr Comp Physiol 2015;308: R1008-R1020.

29. Baker A, Kostov K, Miller R, Weiner M. Slow force recovery after long-duration exercise: metabolic and activation factors in muscle fatigue. J Appl Physiol 1993;74:2294-2294.

30. DeGroot M, Massie BM, Boska M, Gober J, Miller RG, Weiner MW. Dissociation of $\left[\mathrm{H}^{+}\right]$from fatigue in human muscle detected by high time resolution 31P-NMR. Muscle Nerve 1993;16:91-98.

31. Allen DG, Lamb GD, Westerblad H. Skeletal muscle fatigue: cellular mechanisms. Physiol Rev 2008;88:287-332.

32. Metzger JM, Moss RL. pH modulation of the kinetics of a $\mathrm{Ca} 2^{(+)}$-sensitive cross-bridge state transition in mammalian single skeletal muscle fibres. J Physiol 1990;428:751-764.

33. Fernandez-del-Olmo M, Rodriguez F, Marquez G, Iglesias X, Marina $\mathrm{M}$, Benitez A, et al. Isometric knee extensor fatigue following a Wingate test: peripheral and central mechanisms. Scand J Med Sci Sports 2013;23:57-65

34. Bogdanis GC, Nevill ME, Lakomy HKA. Effects of previous dynamic arm exercise on power output during repeated maximal sprint cycling. J Sports Sci 1994;12:363-370.

35. Karlsson J, Bonde-Petersen F, Henriksson J, Knuttgen HG. Effects of previous exercise with arms or legs on metabolism and performance in exhaustive exercise. J Appl Physiol 1975;38:763-767.

36. Baker JS, McCormick MC, Robergs RA. Interaction among skeletal muscle metabolic energy systems during intense exercise. J Nutr Metab 2010;2010:905612.

37. Westerblad H, Allen DG, Lännergren J. Muscle fatigue: lactic acid or inorganic phosphate the major cause? Physiology 2002;17:17-21.

38. Fitts RH. Mechanisms of muscular fatigue. Med Sports Sci 2004;46: 279-300.

39. Bouhlel E, Chelly MS, Gmada N, Tabka Z, Shephard R. Effect of a prior force-velocity test performed with legs on subsequent peak power output measured with arms or vice versa. J Strength Cond Res 2010;24:992-998.

40. Grant MC, Robergs R, Baird MF, Baker JS. The effect of prior upper body exercise on subsequent wingate performance. Biomed Res Int 2014;2014:329328.

41. Duchateau J, Hainaut K. Electrical and mechanical failures during sustained and intermittent contractions in humans. J Appl Physiol $1985 ; 58: 942-947$ 
42. Siegler JC, Marshall P, Pouslen MK, Nielsen NPB, Kennedy D, Green $\mathrm{S}$. The effect of $\mathrm{pH}$ on fatigue during submaximal isometric contractions of the human calf muscle. Eur J Appl Physiol 2015;115:565577.

43. Scherr J, Wolfarth B, Christle JW, Pressler A, Wagenpfeil S, Halle M. Associations between Borg's rating of perceived exertion and physiological measures of exercise intensity. Eur J Appl Physiol 2013; 113:147-155

44. Amann M, Blain GM, Proctor LT, Sebranek JJ, Pegelow DF, Dempsey JA. Group III and IV muscle afferents contribute to ventilatory and cardiovascular response to rhythmic exercise in humans. J Appl Physiol 2010;109:966-976. 